\title{
Laboratory Experiments on Gravity Currents Interacting with Upslope and Overhang Barriers
}

\author{
Maria Rita MAGGI ${ }^{1, \otimes}$, \\ Claudia ADDUCE ${ }^{1}$, and Gregory Francis LANE-SERFF ${ }^{2}$ \\ ${ }^{1}$ Roma Tre University, Rome, Italy \\ ${ }^{2}$ University of Manchester, Manchester, United Kingdom \\ $\triangle$ mariarita.maggi@uniroma3.it
}

\begin{abstract}
The dynamics of steady two-dimensional gravity currents interacting with slopes and overhangs are investigated by laboratory experiments. Parameters such as the initial volume of the dense fluid and the angle of the barrier positioned inside the tank are varied. An image analysis technique is adopted to evaluate the instantaneous density fields. The analysis performed showed how the nature of the barrier affects the dynamics of the dense current.
\end{abstract}

Keywords: gravity currents, laboratory experiments, image analysis technique, complex topography.

\section{INTRODUCTION}

Gravity currents are flows driven by a density difference due to a variation in salinity, temperature or the concentration of suspended particulates. These geophysical flows widely occur spontaneously in nature or for anthropogenic causes (Simpson 1997). Although gravity currents develop generally over more complex floor topographies involving slopes, submarine channels and seamounts (Lane-Serff et al. 1995), most laboratory investigations dealing with the dynamics of those currents considers flows over flat surfaces. These last investigations reveal a lack of knowledge on gravity currents interacting with a complex topography.

The aim of this work is to investigate how even simple variations of the geometry of the domain influence the dynamics of gravity currents by using laboratory experiments. 


\section{EXPERIMENTAL DETAILS}

The laboratory experiments are conducted in a Perspex tank $3 \mathrm{~m}$ long, $0.2 \mathrm{~m}$ wide and $0.3 \mathrm{~m}$ deep. The experimental apparatus (Fig. 1) is similar to that described in Lane-Serff et al. (1995) which allows an incoming steady flow. The lock reservoir is realized with a fixed gate placed at $L_{0}=0.4 \mathrm{~m}$ leaving a rectangular opening at the bottom of the tank. A removable gate covers the opening. A slope is located at $L_{S}=0.85 \mathrm{~m}$ from the fixed gate with different inclination $\theta$, ranging from $15^{\circ}$ and $165^{\circ}$, in order to represent up slopes ( $\mathrm{S}$ runs), or overhanging barriers cases (O runs).

a)

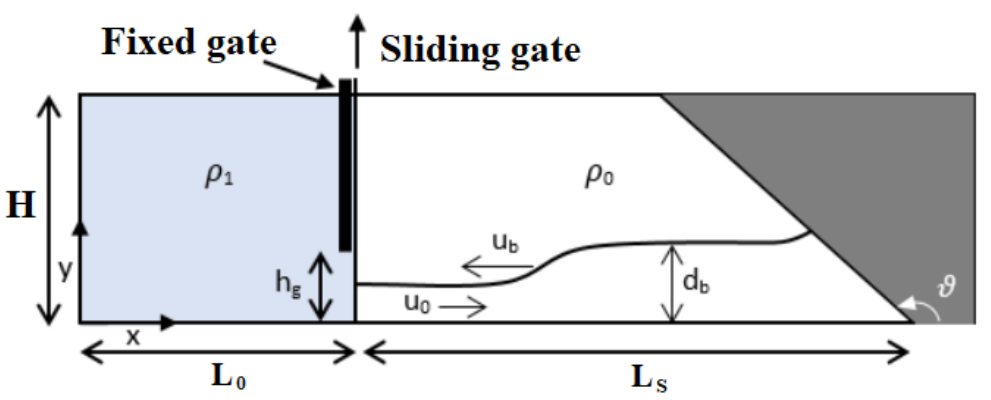

b)

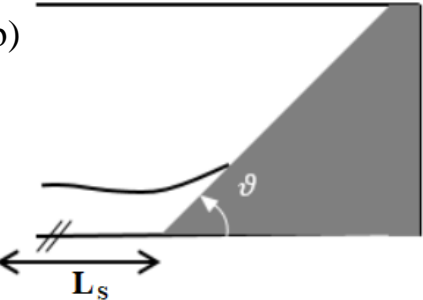

Fig. 1. Sketch of the tank used to perform laboratory experiments. Gravity current reflected back respectively by: a) overhang barrier (O runs); b) slope ( $\mathrm{S}$ runs).

For each experiment the left part of the tank is filled with salty water with initial density $\rho$, while the rest of the tank is filled with an ambient fluid of density $\rho_{0}\left(\Delta \rho=\rho_{1}-\rho_{0}=40 \mathrm{~kg} / \mathrm{m}^{3}\right)$.

A controlled quantity of dye is added to the salty water in order to allow the visualization of the dense fluid. The run starts when the sliding gate is removed and stopped when the reflected dense flow, after the interaction with the slope, reached the position $L_{0}$. The Froude number $F r=U_{b} / \sqrt{g_{0}^{\prime}\left(h_{g} / 2\right)} \cong 0.8$, where $U_{b}$ is the bulk velocity and $g_{0}^{\prime}=g *\left(\Delta \rho / \rho_{0}\right)$ is the initial reduced gravity. The experiments were recorded by a camera with an acquisition frequency of $25 \mathrm{~Hz}$ and spatial resolution of $1024 \times 668$ pixels. Images extracted from the acquired movie were converted into matrices of grey levels. The instantaneous density fields $\rho^{*}(x, y, t)=\left(\rho(x, y, t)-\rho_{0}\right) /\left(\rho_{1}-\rho_{0}\right)$ was obtained by an image analysis technique (Nogueira et al. 2013). The pixel-based analysis of the density fields adopted here allows us to obtain the local density value and then the depth-averaged density, $\bar{\rho}_{v}$. From the $\bar{\rho}_{v}$ is possible to infer the main characteristics of the flow dynamics. Figure 2 shown $\bar{\rho}_{v}$ in the plane $x^{*}-t^{*}$ for the $\mathrm{S}$
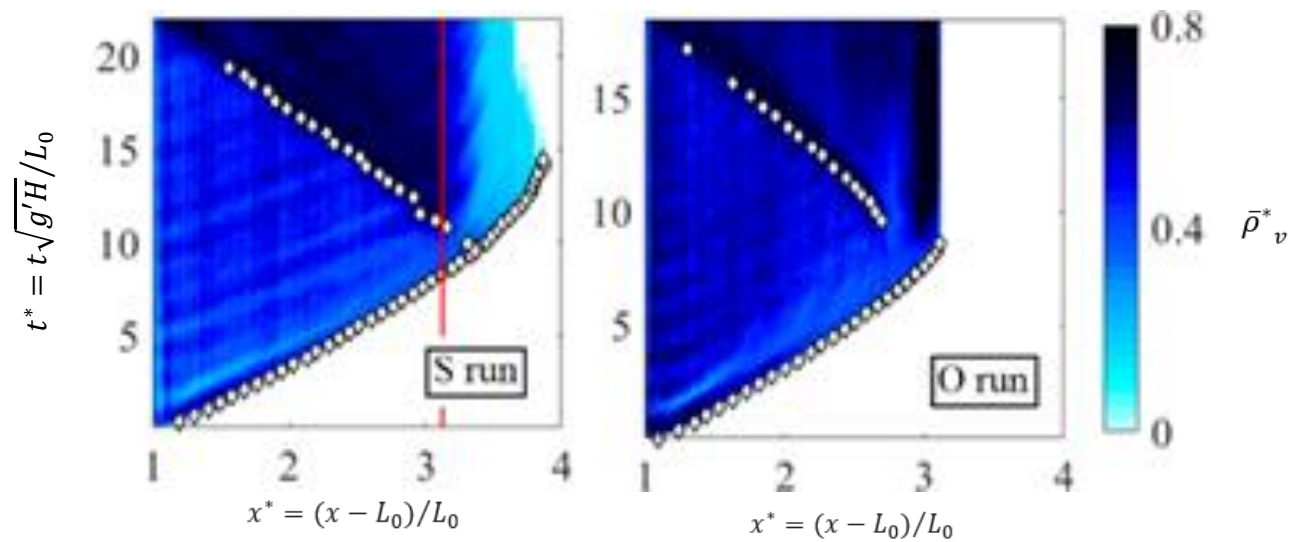

Fig. 2. Dimensionless $\bar{\rho}^{*}$, for the $\mathrm{S}$ run with $\theta=15^{\circ}$ and for the $\mathrm{O}$ run with $\theta=150^{\circ}$. The white markers mark the dimensionless front and reflected bore position. The red line represents the toe of the slope. 
and $\mathrm{O}$ runs. In the up slope case the high values of $\bar{\rho}^{*}{ }_{v}$ are identified in the area near the front of the current and in the outgoing bore, while for the overhang run high values of $\bar{\rho}^{*}{ }_{v}$ can be also observed at the foot of the barrier. The presence of mixing associated with the reflection process is larger for the $\mathrm{O}$ run than the $\mathrm{S}$ run. The density fields emphasizes a more complex dynamics in the $\mathrm{O}$ run related to the presence of the barrier even before the current interacts with it.

\section{References}

Lane-Serff, G.F., L.M. Beal and T.D. Hadfield (1995), Gravity current flow over obstacles, J. Fluid Mech. 292, 39-53, DOI: 10.1017/S002211209500142X.

Nogueira, H.I.S., C. Adduce, E. Alves, and M.J. Franca (2013), Image analysis technique applied to lock-exchange gravity currents, Meas. Sci. Technol. 24, 4, 047001.

Simpson, J.E. (1997), Gravity Currents: In the Environment and the Laboratory, 2nd ed., Cambridge University Press, Cambridge, 258 pp. 